\title{
Viewpoint
}

\section{Divided rheumatological care: the advent of the nurse practitioner?}

The rheumatological clinics at the General Infirmary at Leeds are moving towards a divided system of patient care. This change was initiated to fulfil a particular local need and may not necessarily be applicable to other rheumatology units. Nevertheless it continues to evolve and adapt in accordance with needs, and individual rheumatologists elsewhere in the United Kingdom may spot aspects of the system that would be appropriate to their own particular circumstances.

There are relatively fewer rheumatologists in Yorkshire than in other parts of the United Kingdom. The long-term aim should be to fight for more at a local level, but in the short term, in order to keep pace with demand, rheumatologists have to choose between providing comprehensive care for a few (restricting access to clinics) or spreading the care thinly across clinics of unrestricted access. A third possible solution evolved. This was to seek independent short-term financing and develop a system of care to match the needs-surely preferable to wringing one's hands in despair and handing around the Valium.

By the conversion of examination rooms into consulting rooms the Outpatient Department at the General Infirmary at Leeds is easily adapted to providing (rather cramped) facilities for more people to see more patients. These extra rooms have been exploited by the research nursing sisters that have been employed in Leeds for 9 years. Their role has recently been further expanded into that of 'clinical metrologists' ${ }^{1}$ with the establishment of a Clinical Pharmacology Unit and the need to use these nursing sisters to see and assess a large number of patients participating in clinical trials. They have thus conducted booked clinics devoted to the care of trial patients in rooms adjacent to the consulting physicians and junior hospital staff for several years.

We may have been lucky in that a reluctance by local Health Service administrators to employ nurses of sister grade on a part-time basis allowed us a choice from an average of 25 highly qualified applicants for every part-time university nurse's post advertised in the Leeds area. This allowed the selection of very able applicants, who soon came to appreciate, like many doctors before them, that the rigorous measurement of routine trials contributed only a small part of total patient care.

The progression was inevitable. Physicians recruited more and more patients to clinical trials. This reflected a healthy interest in research and a natural desire to have groups of patients (ankylosing spondylitics, 'early rheumatoids,' and so on) closely monitored while taking appropriate therapy in the belief that patients participating in clinical trials are receiving the best and most careful continuous assessment. With the clinical trials largely conducted by metrologists, physicians were left free to concentrate on the art of differential diagnosis and to provide a service to more patients. Subsequently patients tended to prefer a series of appointments with a research nurse than a more cursory visit to the rheumatologists-a chastening thought for the physician concerned. The reason is clear. A 15-minute booked appointment with a nurse to allow time for her to perform the articular index and other assessments allowed the patient access to 15 minutes of paramedical time in which to present important questions on topics such as footwear, exercise, and splinting. The 'nurse practitioner' of transatlantic fame had arrived in the United Kingdom. True an evaluation of the nurse in imparting surgical information ${ }^{2}$ has suggested that patients prefer to talk to surgeons rather than nurses, but our anecdotal experience (at present under critical evaluation) suggests this is not usually so in the field of chronic disabling diseases.

Just as the general practitioner may utilise a district nurse to provide a 2-tier system of care in the community, so a comparable hospital system of 2-tier care with nurse practitioners consulting with patients in rooms adjacent to rheumatologists is almost upon us in some United Kingdom centres. The paramedical workers (nurses, physiotherapists, occupational therapists-what happened to the poor old ward pharmacists?) will convene in parallel with the Heberden Society and the BARR in 1983, as has occurred in America for many years. Now is the time to temper enthusiasm with a pause for reflection.

A divided system of hospital care certainly merits urgent evaluation in a variety of specialties; nurse practitioners may be as relevant to the management of diabetics and respiratory cripples as they are to 
rheumatic patients. Clinical pharmacologists may also find them of value. A further role for nurse practitioners is in the management of second-line drug clinics in the rheumatic diseases: society did not train a rhe umatologist for 14 years in order to have him check platelet counts. Patient education is another important field of activity.

One criticism levelled against this 2-tier arrangement is that the lower tier provides an inferior level of patient care. In our experience the care is complementary, but, as a safeguard, patients booked to see the nurse practitioner are switched at intervals to the physician's clinic and vice versa. In the USA the 2 levels have sometimes gone their own ways. At the paramedical sessions of the American Rheumatism Association's meetings (rarely attended by physicians) enthusiasm may replace objective data, and controlled work is hard to find. Early critical assessment is required.

The paramedicals seem unlikely to supplant the surfeit of young doctors emerging from medical schools. They will complement them and leave doctors free to direct and co-ordinate treatment. The shorter period of paramedical training also ensures that physicians with their wider expertise will still need to integrate the different health professionals in rehabilitation work, which, to its benefit, becomes increasingly scientific. By contrast general practitioner clinical assistants may feel threatened-a well trained nearly full-time nurse may contribute more to a hospital department than a very part-time doctor. Much as one would like to allow general practice a foothold in the hospital, the rational step may be to push general practice rheumatological care back into general practice. Recruitment of patients to studies of gout and low back pain becomes increas- ingly hard in hospitals as these conditions become more and more the province of the general practitioner.

Rheumatologists may also feel threatened, with some reason, when a nurse practitioner takes over much of their educative function. Urgent action should defuse the situation. Nurses are a much more heterogeneous population, both in experience and in basic qualifications, than doctors. Some of them will aspire to higher things and should be encouraged to do so; one suspects that they are needed as much as the physicians by the patients. Potential nurse practitioners may wish to consider unhitching themselves from the conventional nursing bureaucracy; appointments committees dominated by nursing administrators simply breed more generations of nursing administrators. Physicians for their part should encourage paramedical high fliers by seeking soft funds (in the first instance) to make nurse practitioner or comparable appointments, supervising the selection so that appointees have the background and confidence to complement the physician's skills, and then training them to an appropriate high standard (the deficiency of an appropriate textbook is shortly to be remedied) while evaluating their performance in their new career grade.

H. A. BIRD

General Infirmary at Leeds

\section{References}

1 Bird H A, Wright V, Galloway D. Clinical metrology-a future career grade? Lancet 1980; ii: 138-9.

2 French K. Some anxieties of elective surgery patients and the design for reassurance and information. Res Psychol Med 1979; 2: 336-43. 